\title{
Power Density Case Study for 5G mmWave Array Antennas
}

\author{
Dianyuan Qi $\mathbb{D}^{1},{ }^{1}$ Fangzhu Zou $\mathbb{D}^{1},{ }^{1}$ Jing Zhao $\mathbb{D}^{1},{ }^{1}$ Shaobin Sun $\mathbb{D}^{2},{ }^{2}$ Huanbin Wei $\mathbb{D}^{2}$ \\ Yiling Chen $\mathbb{D}^{2}$, and Zhan Xia $\mathbb{D}^{2}$ \\ ${ }^{1}$ China Academy of Information and Communications Technology, Beijing 100191, China \\ ${ }^{2}$ Huawei, Shenzhen 518129, China
}

Correspondence should be addressed to Fangzhu Zou; zoufangzhu@caict.ac.cn

Received 25 January 2021; Revised 31 March 2021; Accepted 13 May 2021; Published 9 June 2021

Academic Editor: Yuanpeng Zhang

Copyright (C) 2021 Dianyuan Qi et al. This is an open access article distributed under the Creative Commons Attribution License, which permits unrestricted use, distribution, and reproduction in any medium, provided the original work is properly cited.

\begin{abstract}
As 5G millimeter wave (mmWave) wireless device involves some new technologies, such as beamforming, the radiofrequency (RF) exposure compliance test for the $5 \mathrm{G}$ mmWave wireless device is significantly complicated. In order to shorten the compliance period for $5 \mathrm{G}$ mmWave terminals, the relevant regulatory authorities recommend a combination of numerical simulation and measurements to demonstrate compliance. To verify the feasibility of this method, the RF exposure test conducted in this paper was a reverse procedure according to IEEE (the Institute of Electrical and Electronics Engineers) standards. First, actual measurements under various conditions, including different beam configurations, different test distances, different input power levels, different duty cycle, and nonpeak directions, were performed, and the changing trend of PD over testing conditions was analyzed. Then one dual-polarized patch antenna array was selected for simulation analysis. The feasibility of the method proposed in IEEE standards was proved through the comparison of the results experiment and numerical analysis.
\end{abstract}

\section{Introduction}

The 3rd Generation Partnership Project (3gpp) protocol divides the $5 \mathrm{G}$ frequency spectrum into two FR (Frequency Range). FR1 is a low-frequency band up to $7125 \mathrm{MHz}$, and FR2 ranges from $24.25 \mathrm{GHz}$ to $52.6 \mathrm{GHz}$. Most of the electromagnetic wavelengths in this FR2 are in the millimeter level. As mmWave technology can achieve a faster speed and lesser extent latency than $4 \mathrm{G}$ network, 5G wireless device has attracted massive attention in recent years. In the meantime, it also has raised the public concern regarding the biological effects on the human body resulted from the exposure to such high-frequency electromagnetic field (EMF). To control the effect in a safe range, standard organizations such as the International Commission for the Protection of NonIonizing Radiation (ICNIRP) and IEEE have established the permissible exposure limits for electromagnetic radiation [1].

Specific absorption rate (SAR) and power density (PD) are usually adopted to determine exposure compliance. SAR defines the amount of energy absorbed by human tissue per mass unit, and it is currently preferred in exposure com- pliance determination. However, when higher frequencies are involved, the skin depth decreases, and energy absorption is usually limited to the skin surface. Studies have shown that when the frequencies are at $10 \mathrm{GHz}-100 \mathrm{GHz}$, about $90 \%$ of the energy will be absorbed by the skin's epidermis or dermis [2]. In such circumstance, it is difficult to build a meaningful volume for SAR. The mmWave devices operating at such high-frequency range should be evaluated with power density, as it is expressed per unit area for near-field RF exposure evaluation, instead of per unit mass in SAR. Whereas power density measurement is a time-consuming procedure; therefore, combining simulation and measurement has been proposed as an efficient method during a compliance test.

\section{Device under Test}

The device used in this testing is a prototype board that operates at $28 \mathrm{GHz}$, and the bandwidth is $200 \mathrm{MHz}$. There are five $5 \mathrm{G}$ antenna arrays at different sides of the device as shown in Figure 1. The details of the device antenna are as follows: 


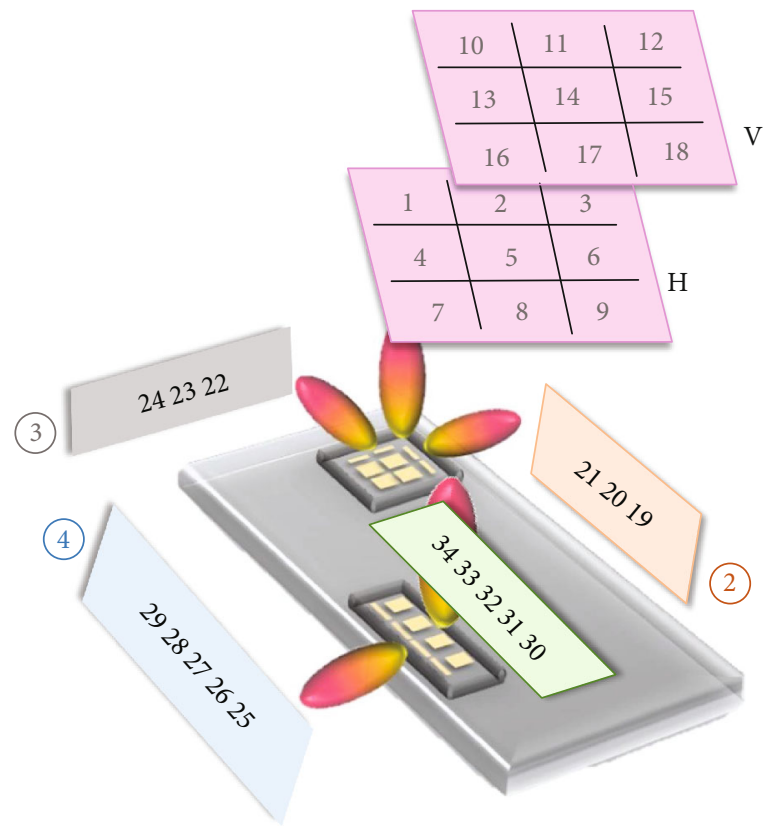

FIgURE 1: Antenna location and beam configuration (rear view).

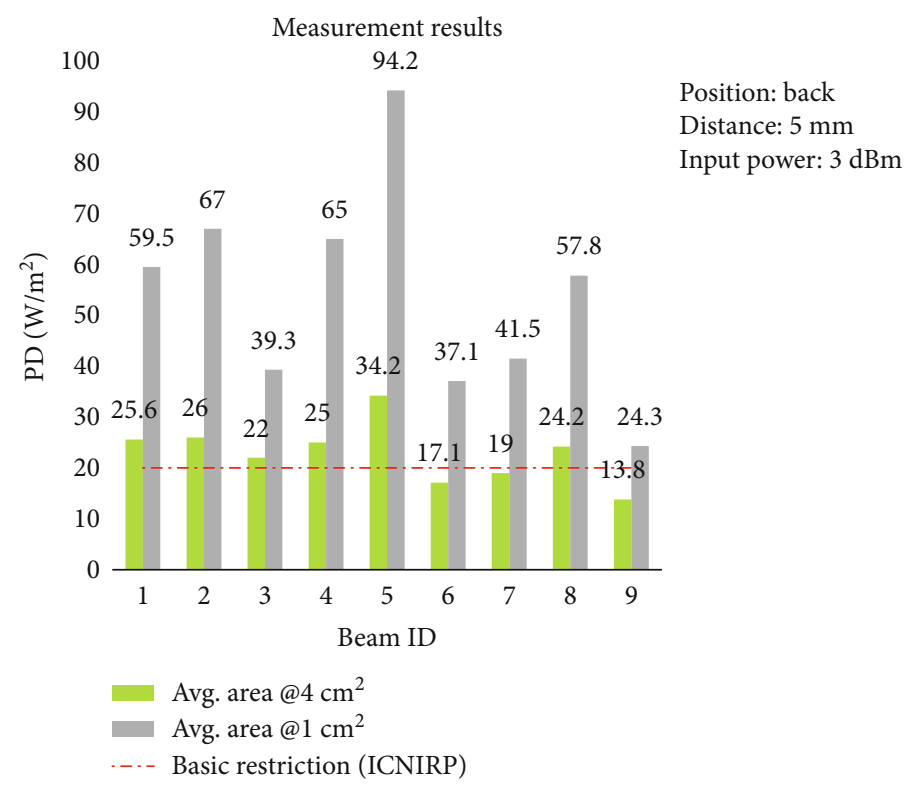

Figure 2: Measurement results of beam ID 1-9.

(1) One $2 \times 2$ patch antenna array, dual-polarized, is located at the left corner of DUT. Beam 1-18 are generated by this dual-polarized patch antenna, of which beam 1-9 are horizontal polarization and beam 10-18 are vertically polarized

(2) Two $1 \times 2$ dipole antenna arrays, single-polarized, are located at the left corner of the device. Beam 19-20 are generated by the antenna at the left edge. Beam 22-24 are generated by the antenna at the top edge

(3) One $1 \times 4$ dipole antenna array, single-polarized, is located at the right edge of the device. Beam 25-29 are generated by this antenna
(4) One $1 \times 4$ patch antenna array, dual-polarized, is located at the right edge of DUT. Beam 30-34 are generated by this patch antenna

The antenna arrays cannot work simultaneously. While one antenna is active, the rest of the arrays will be disabled. As for beamforming, the beam configuration of an antenna array can be changed by software.

\section{Power Density Assessment}

The power density testing employs the mmWave module of cDASY6 from SPEAG, Switzerland. The mmWave 


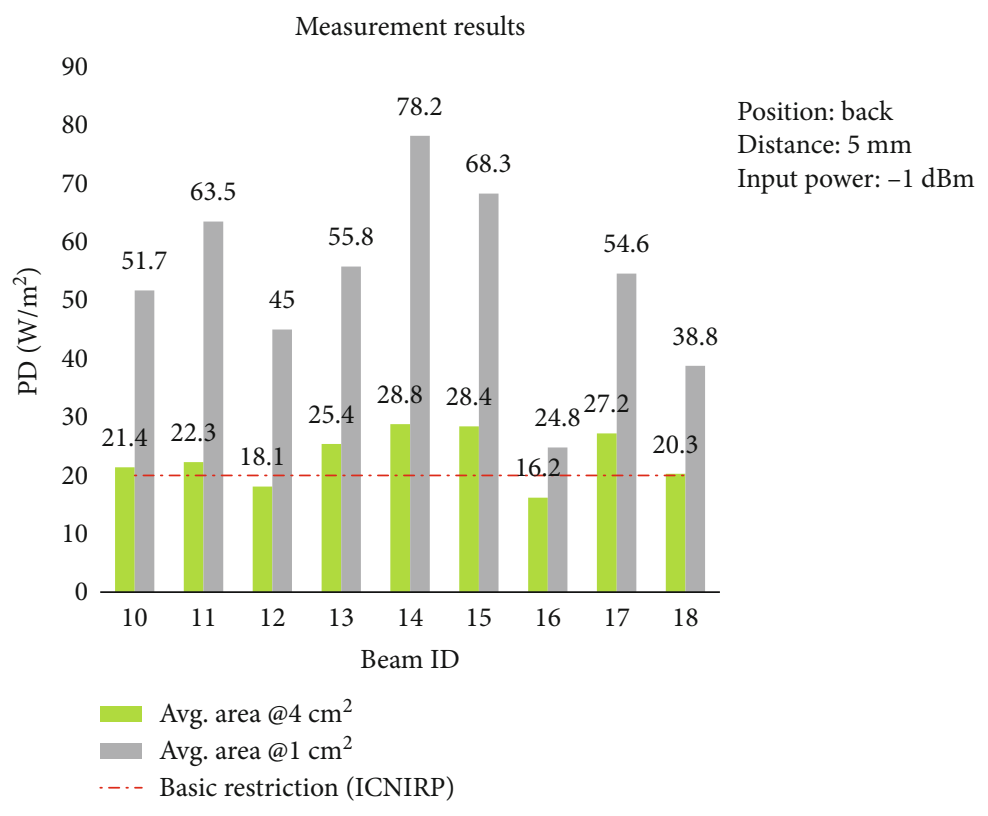

Figure 3: Measurement results of beam ID 10-18.

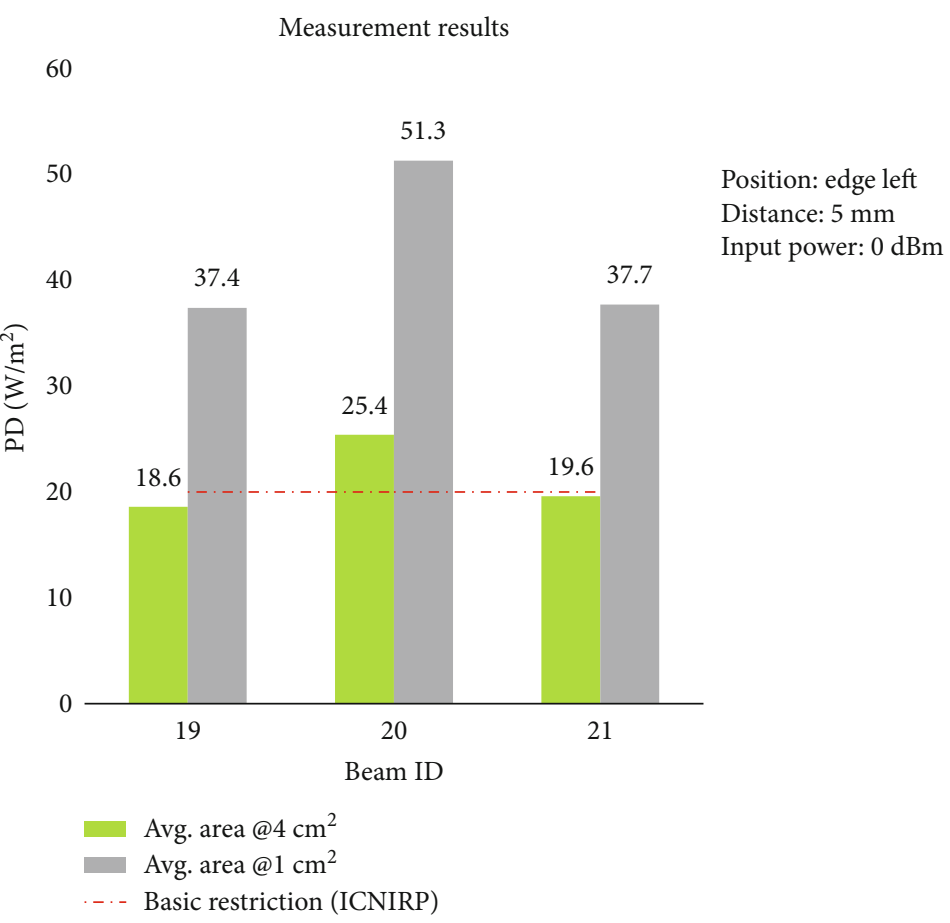

Figure 4: Measurement results of beam ID 19-21.

module supports a measurement at distances as small as $2 \mathrm{~mm}$ and to automatically determine the optimal measurement grid based on the frequency modulation, evaluation distance, and extent of the transmitter array.

The phantom of this mmWave module approximates free-space conditions and allows evaluating not only the antenna side of the device under test (DUT) but also any opposite-radiating side of the devices operating above $10 \mathrm{GHz}$.
The probe used in the measurement is an E-field mmWave probe. It can perform at the frequency range from $750 \mathrm{MHz}$ to $110 \mathrm{GHz}$. According to the current draft of IEC/IEEE 63195, for general near-field measurements, the magnitudes and phases of both E-filed and $\mathrm{H}$-field are necessary to correctly determine the power density. As an E-field probe is adopted during measurement, the field reconstruction is used to derive the $\mathrm{H}$-field from the direct measurement of E-field. All components of the E-field, the H-field, 


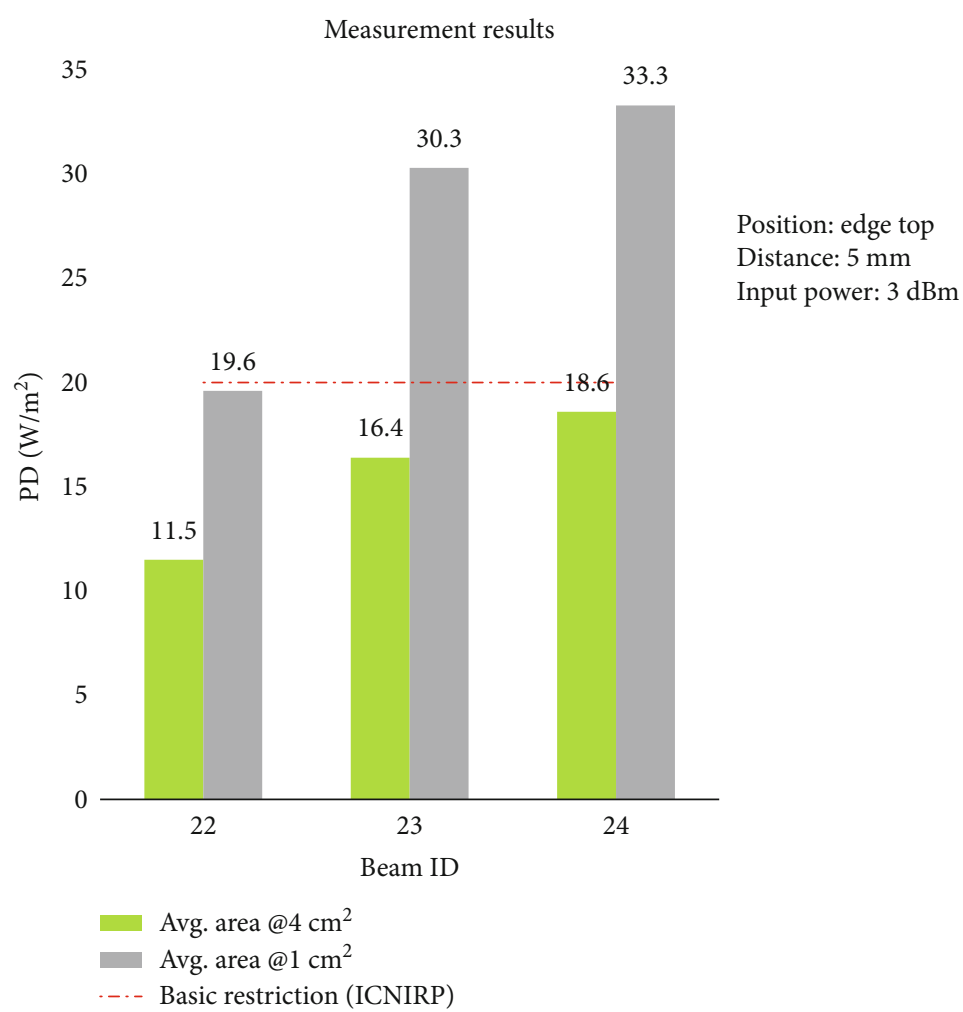

Figure 5: Measurement results of beam ID 22-24.

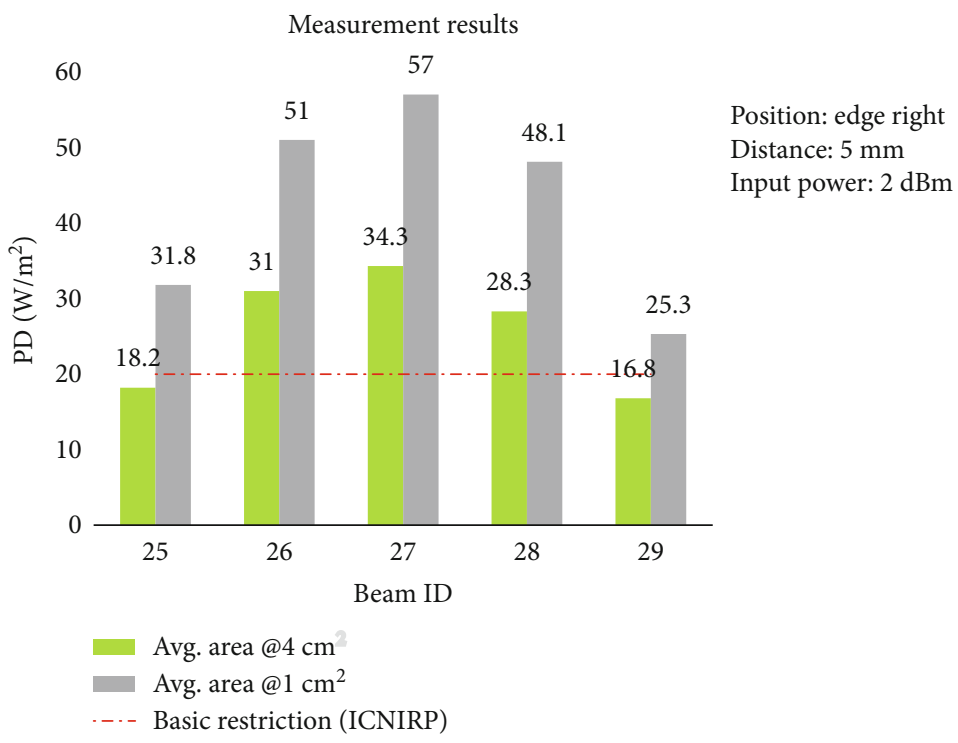

FIgURE 6: Measurement results of beam ID 25-29.

and the power density on the measurement plane are reconstructed through an approach based on the Gerchberg-Saxton algorithm with the mmWave module of cDASY6 $[2,3]$.

\section{Test Settings}

During the power density measurement, the mmWave signal in the DUT is configured by an engineering testing software, and the test mode is nonsignaling. This testing is implemented in 5 different situations:

(1) The device is operated to transmit continuously at $100 \%$ transmission duty with different antenna beams. The test distance is $5 \mathrm{~mm}$, and the input power is specified for each antenna array

(2) For certain beams, 3 different test distances are set from $10 \mathrm{~mm}$ to $20 \mathrm{~mm}$ with $5 \mathrm{~mm}$ increments. The 


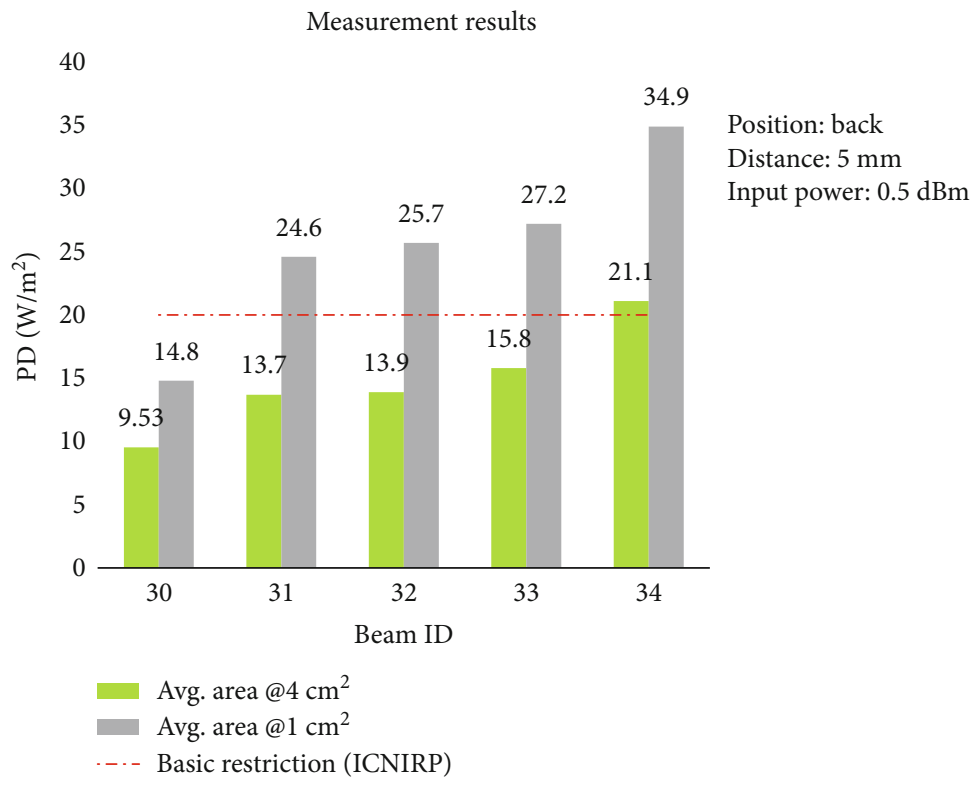

Figure 7: Measurement results of beam ID 30-34.

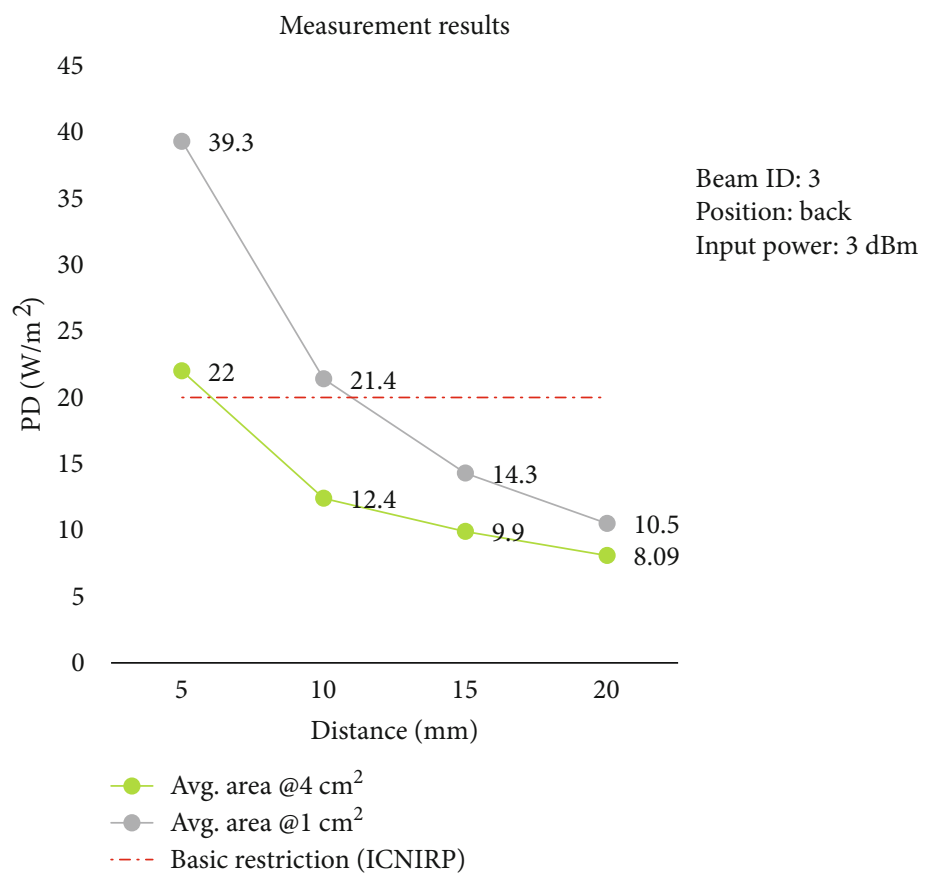

Figure 8: Measurement results at different test distances for beam 3.

device is operated to transmit continuously at $100 \%$ transmission duty, and the input power is specified for each antenna array

(3) Each measurement of certain beams is performed with different input power levels and at $100 \%$ transmission duty. The test distance is $5 \mathrm{~mm}$

(4) The measurements of a certain beam are implemented in duty cycle of $20 \%$ and $60 \%$, respectively, with $5 \mathrm{~mm}$ test distance
(5) The measurements are implemented on the nonpeak directions of certain beams with specified input powers and $5 \mathrm{~mm}$ test distance

\section{Measurement Results}

The PD shown as follows is computed under the averaging area of $1 \mathrm{~cm}^{2}$ and $4 \mathrm{~cm}^{2}$. As the antenna arrays operate at $28 \mathrm{GHz}$, according to ICNIRP RF Guidelines, the data of concern is averaged over $4 \mathrm{~cm}^{2}$ in this paper. 


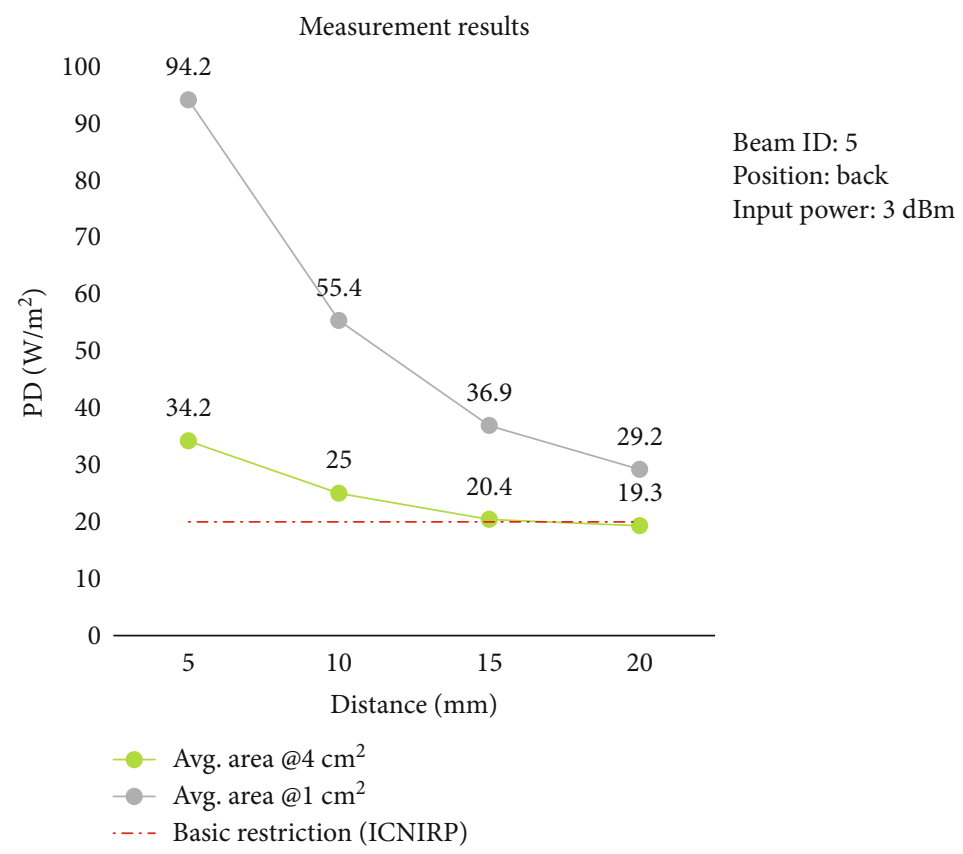

FIGURE 9: Measurement results at different test distances for beam 5.

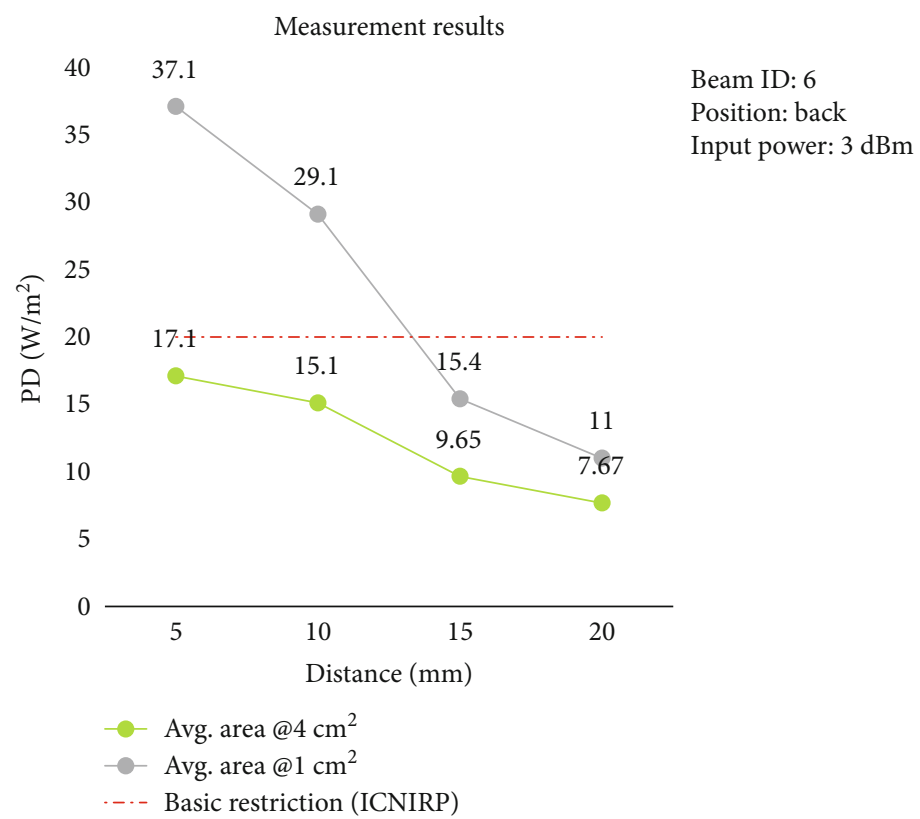

Figure 10: Measurement results at different test distances for beam 6 .

5.1. Power Density Measurement on Different Beam Configurations. As shown in Figures 2 and 3, the highest measured power density beam ID is 5 at $34.2 \mathrm{~W} / \mathrm{m}^{2}$, and beam 14 is the maximum measured beam (power density of $28.8 \mathrm{~W} / \mathrm{m}^{2}$ ), which are the central vectors for their respective polarizations. Moreover, the $\mathrm{H}$ polarization beams of this $2 \times 2$ patch antenna have higher power density than its $\mathrm{V}$ polarization beams. Most of the values obtained from this antenna array are above the basic restriction of $20 \mathrm{~W} / \mathrm{m}^{2}$ as averaged over $4 \mathrm{~cm}^{2}$ according to ICNIRP.

Most of the beams of the single-polarized antenna array at the left edge are below the exposure limits, and the maximum power density is $25.4 \mathrm{~W} / \mathrm{m}^{2}$ found from beam 20 (as shown in Figure 4).

Results in Figure 5 demonstrate that the PD of the antenna at the top edge does not exceed the current ICNIRP basic limit of $20 \mathrm{~W} / \mathrm{m}^{2}$ at $4 \mathrm{~cm}^{2}$. 


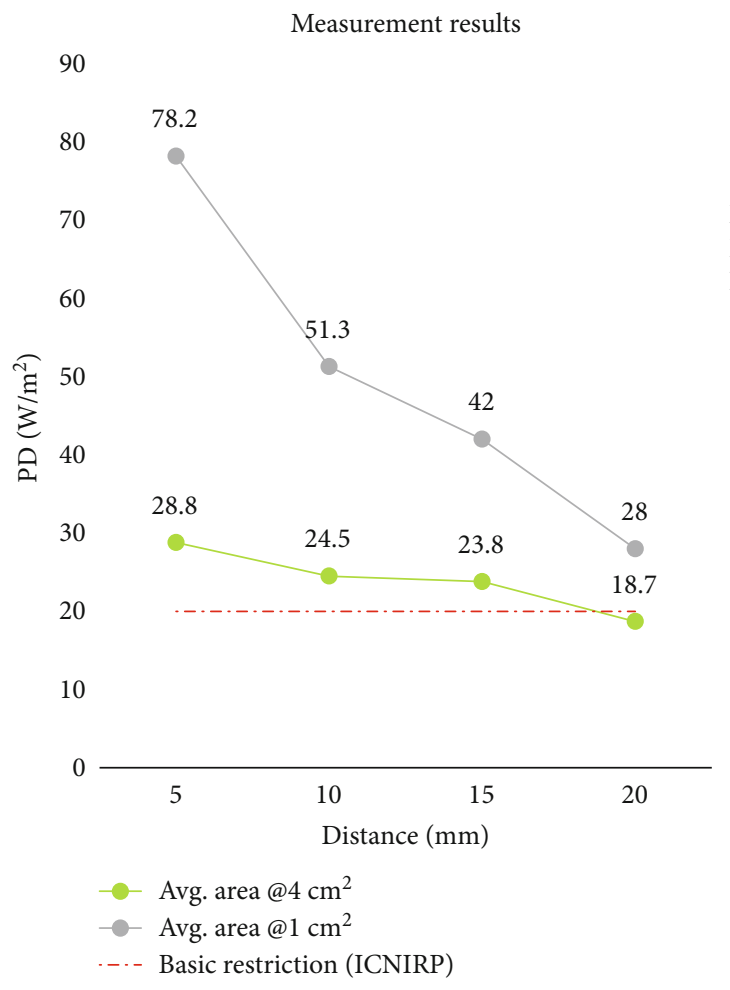

Figure 11: Measurement results at different test distances for beam 14.

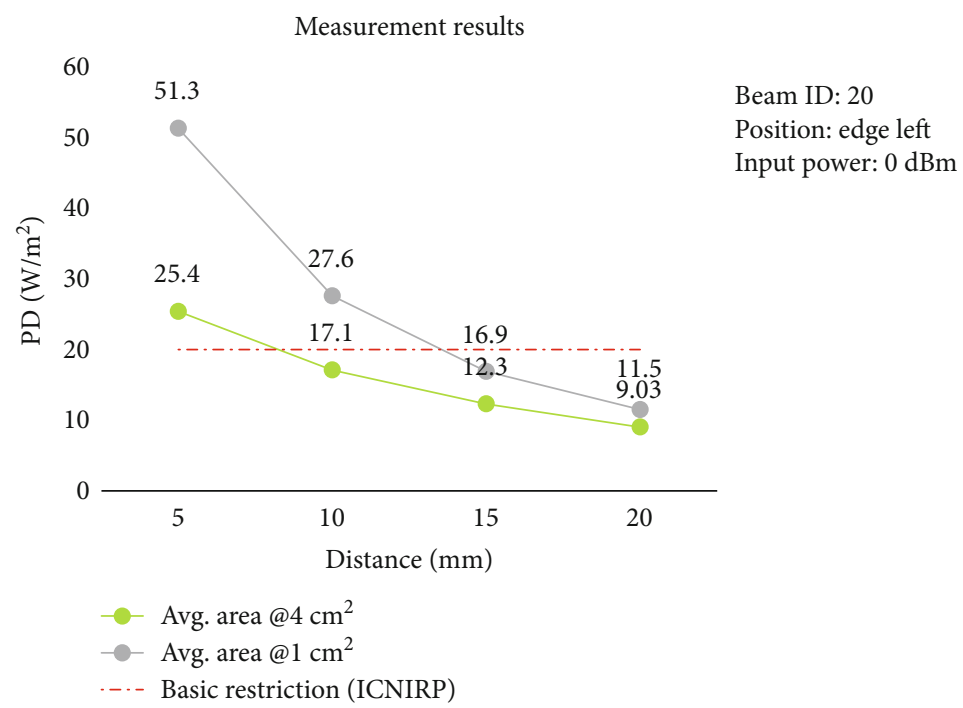

FIgURE 12: Measurement results at different test distances for beam 20.

The results shown in Figure 6 are measured from the single-polarized antenna array at the right edge. Out of the total five beams, three beams are larger than $20 \mathrm{~W} / \mathrm{m}^{2}$. The largest value is measured from beam 27 at $34.3 \mathrm{~W} / \mathrm{m}^{2}$.

Figure 7 is the measured results of the $1 \times 4$ patch antenna array beams. They indicate that beam 30-33 comply with the RF radiation exposure limits of the ICNIRP.
ID: 14

Position: back

Input power: $-1 \mathrm{dBm}$ 


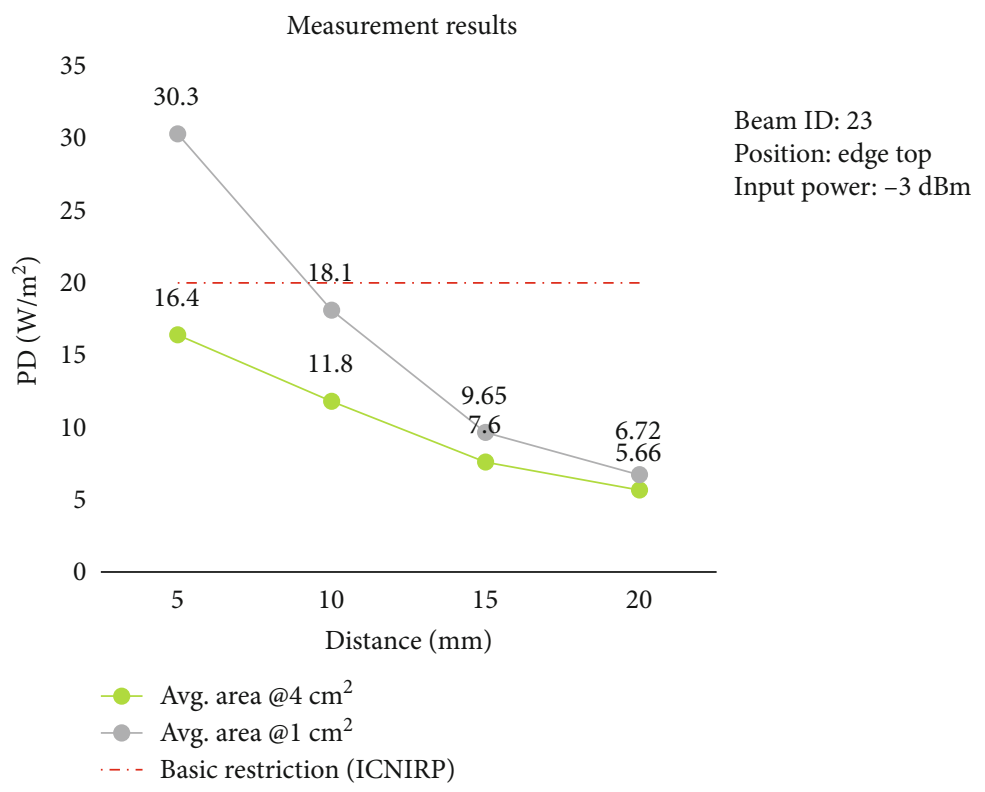

Figure 13: Measurement results at different test distances for beam 23.

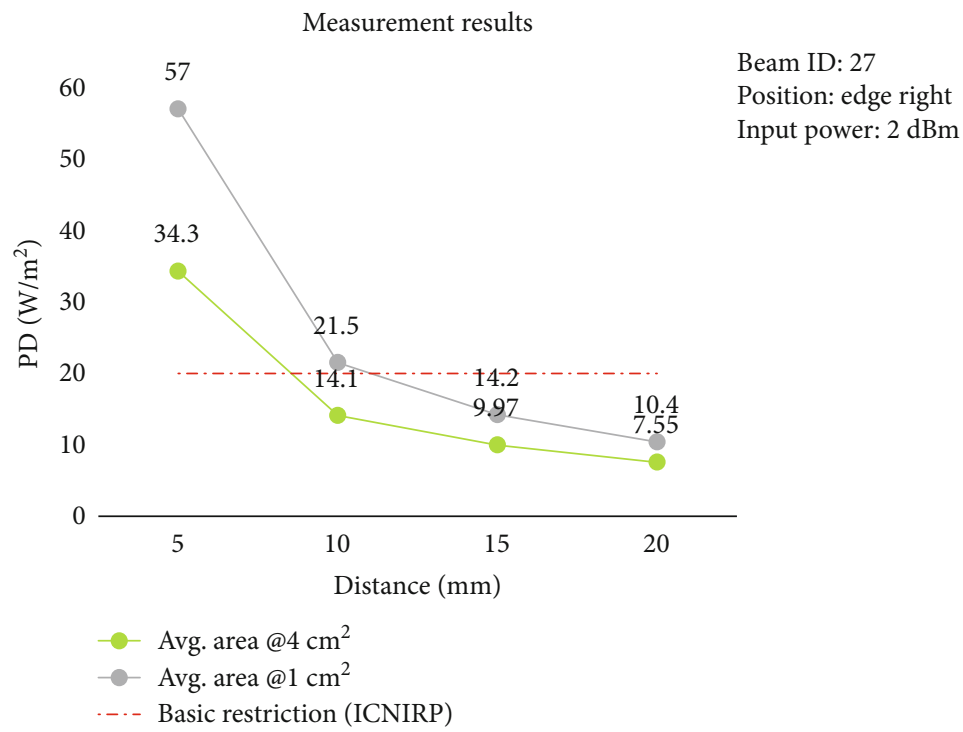

FIGURE 14: Measurement results at different test distances for beam 27.

5.3. Power Density Measurements on Different Input Power. Figures 16 and 17 show that the measurement values go down when the input power levels are reduced by $-3 \mathrm{~dB}$ and $-5 \mathrm{~dB}$, respectively, for both beam 14 and 23 . The power density of beam 14 is finally below $20 \mathrm{~W} / \mathrm{m}^{2}$ at $4 \mathrm{~cm}^{2}$ as the input power is decreased from $-1 \mathrm{~dB}$ to $-4 \mathrm{~dB}$.

5.4. Power Density Measurements under Different Duty Cycle. As shown in Figure 18, there is a significant trend in the measurement result change under different duty cycle, i.e., when the duty cycle is 0.6 , the measured power density is approxi- mately $60 \%$ of that at its maximum duty cycle, and the same is true when the duty cycle is $20 \%$.

5.5. Power Density Measurements at the Nonpeak Direction. Beam 20 is generated by the single-polarized dipole antenna located at the left corner. Figure 19 shows that the power density at the back (nonpeak direction) is close to the highest simulated power density at the left edge, while the power density of beam 23 generated by the left corner antenna at the back (nonpeak direction) is approximately equal to the highest power density at the top edge (as shown in Figure 20). 


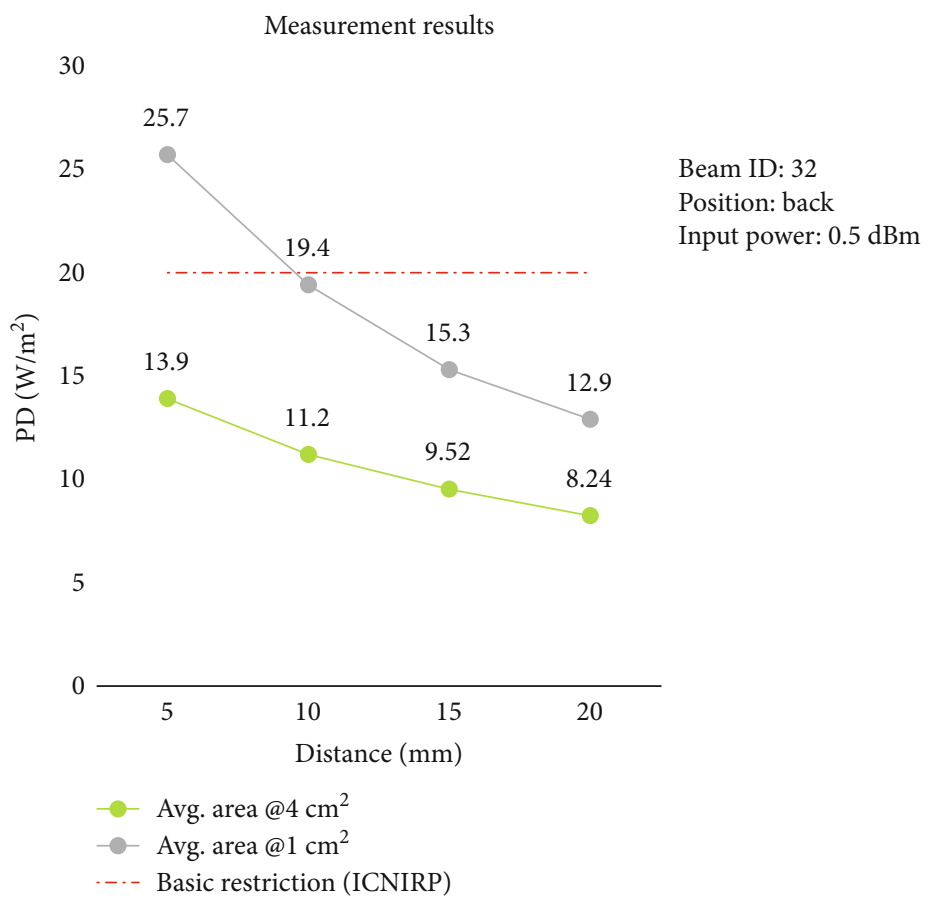

FIGURE 15: Measurement results at different test distances for beam 32.

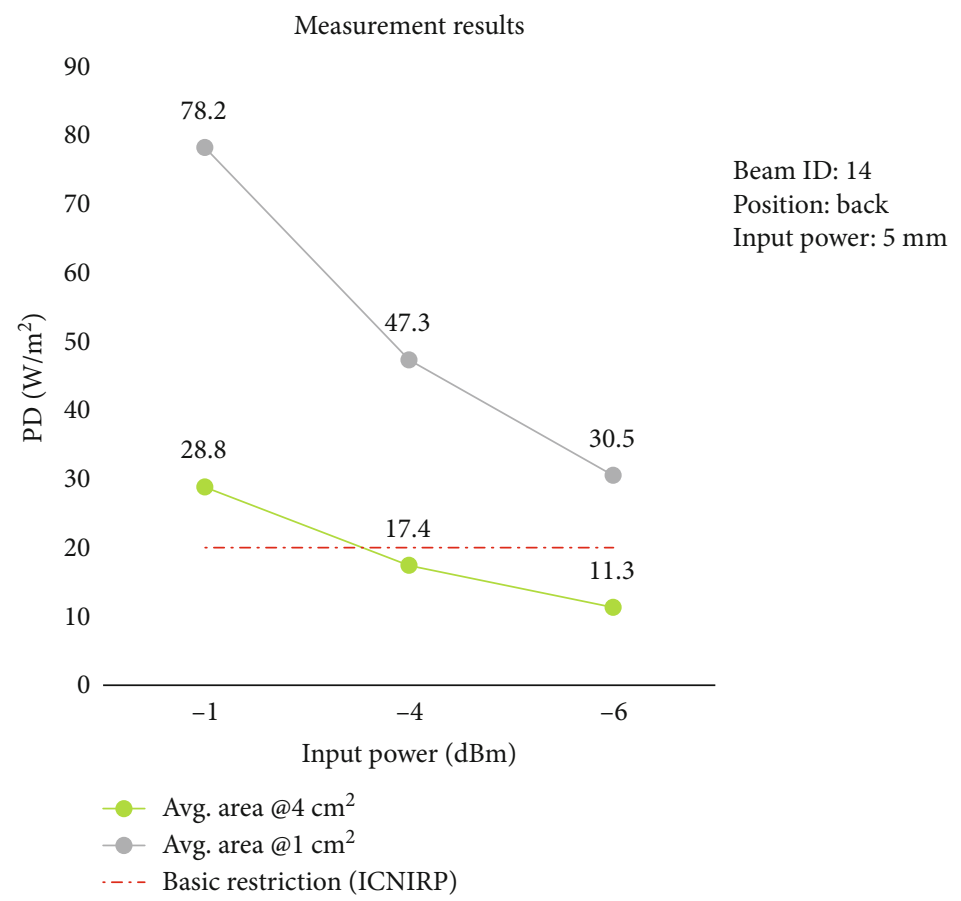

FIGURE 16: Measurement results on different input power for beam 14.

Beam 2, beam 6 , and beam 8 are generated by $2 \times 2$ patch antenna array that is located at the back. As can be seen from Figures 21-23, the measured power density of each beam at the nonpeak directions is much less than that on the major radiation direction.

\section{Simulation and Measurement Results Comparison}

6.1. Simulation Environment. For providing case study for power density analysis, we perform simulation and 


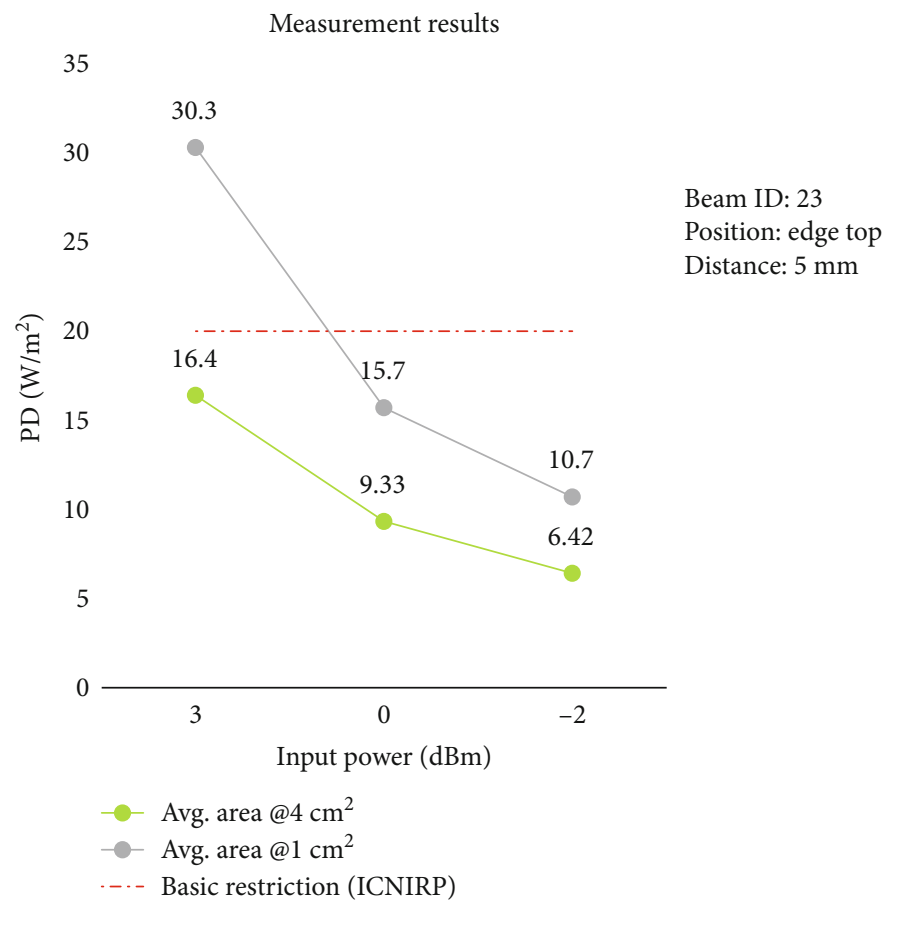

FiguRE 17: Measurement results on different input power for beam 23.

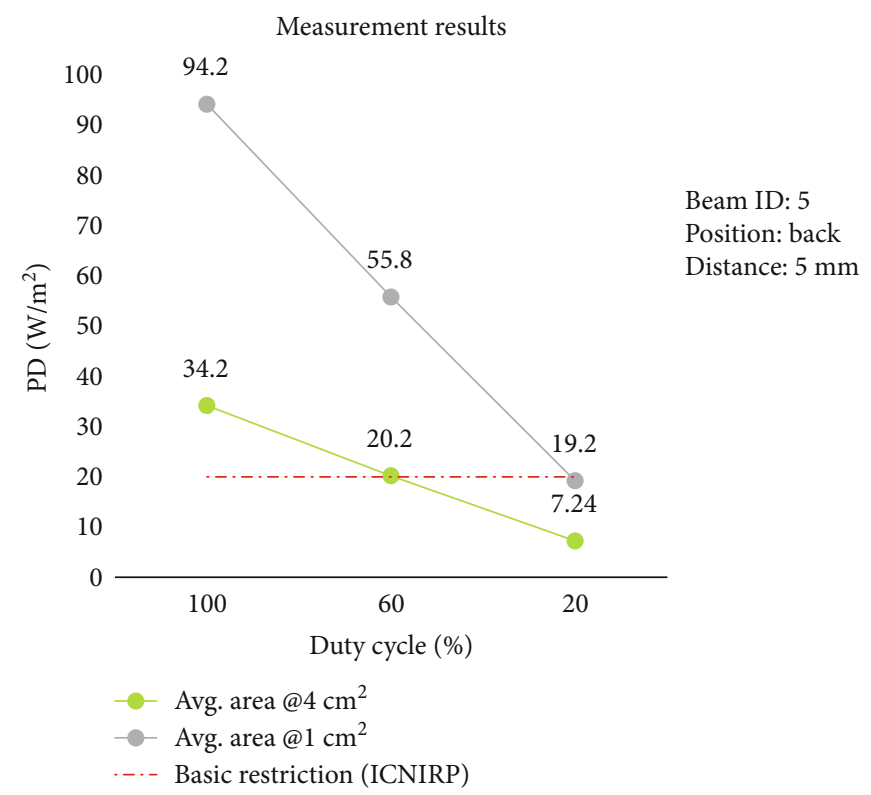

FiguRe 18: Measurement results under different duty cycle for beam 5 .

measurement comparison for considered array antenna. The commercial simulation tool CST based on FDTD (Finite-Difference Time-Domain) algorithm is used for power density simulation.

To explore the consistency of simulation and measurement, the simulated results of $2 \times 2$ patch antenna array will be discussed.

6.2. Power Density Simulation Results. In order to save computational resources, the DUT model is truncated and only the $2 \times 2$ patch antenna array located at the left corner is modeled and simulated in this paper.

The detailed simulation results of the selected $2 \times 2$ patch antenna array beams are shown in Table 1. The DUT is modeled in free-space and the same configurations as the (1) testing condition mentioned in chapter 4 , where the averaging area is $4 \mathrm{~cm}^{2}$ and distance is $5 \mathrm{~mm}$ from the back side.

The simulation results have been normalized to the same PA output power level for comparison with the measurement 


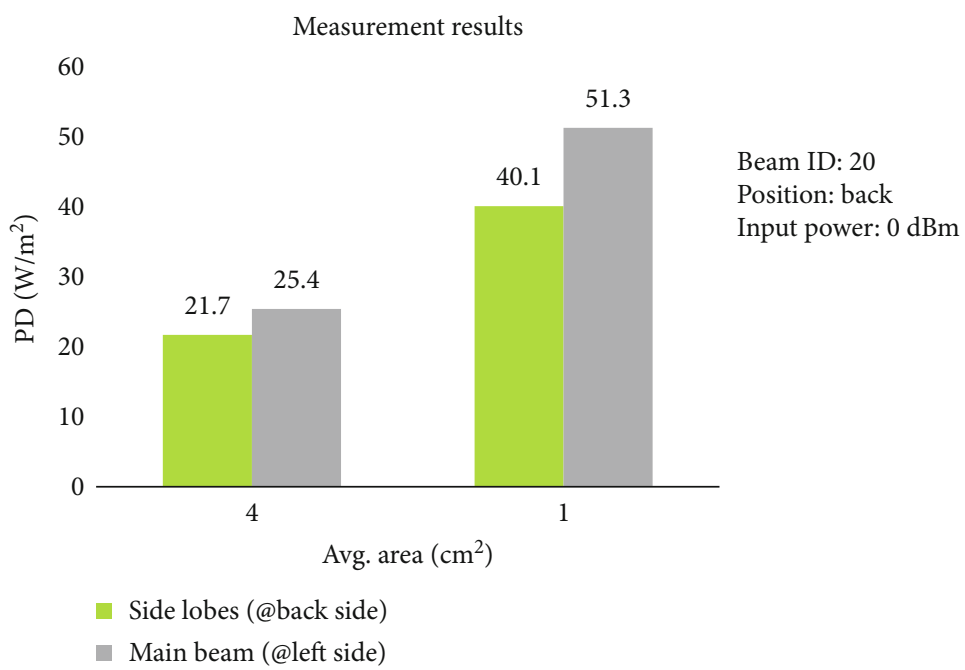

FIGURE 19: Measurement results in a nonpeak direction for beam 20.

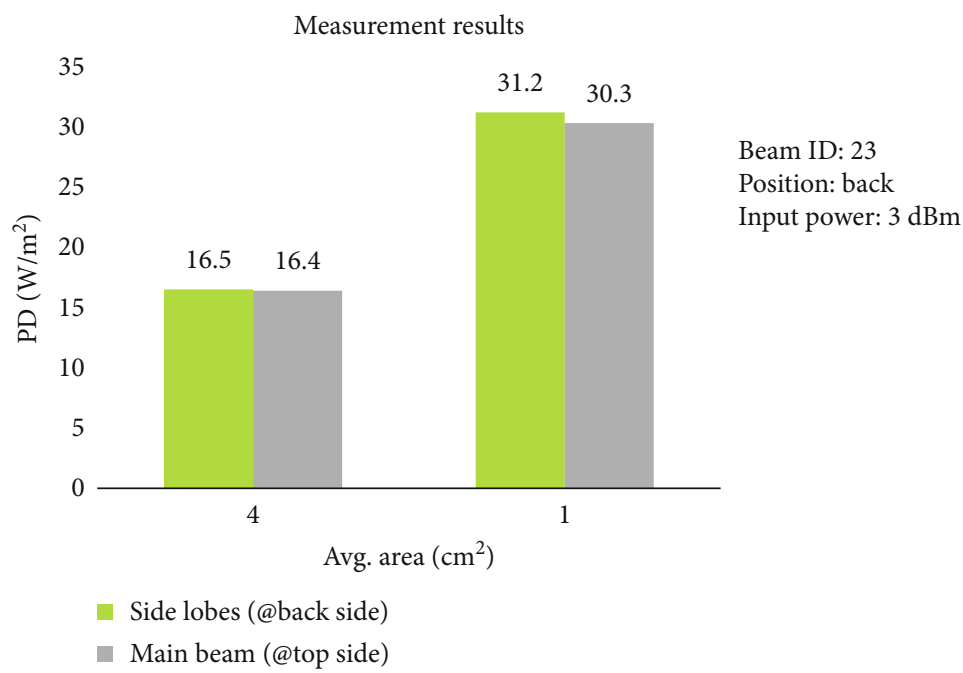

FIGURE 20: Measurement results in a nonpeak direction for beam 23.

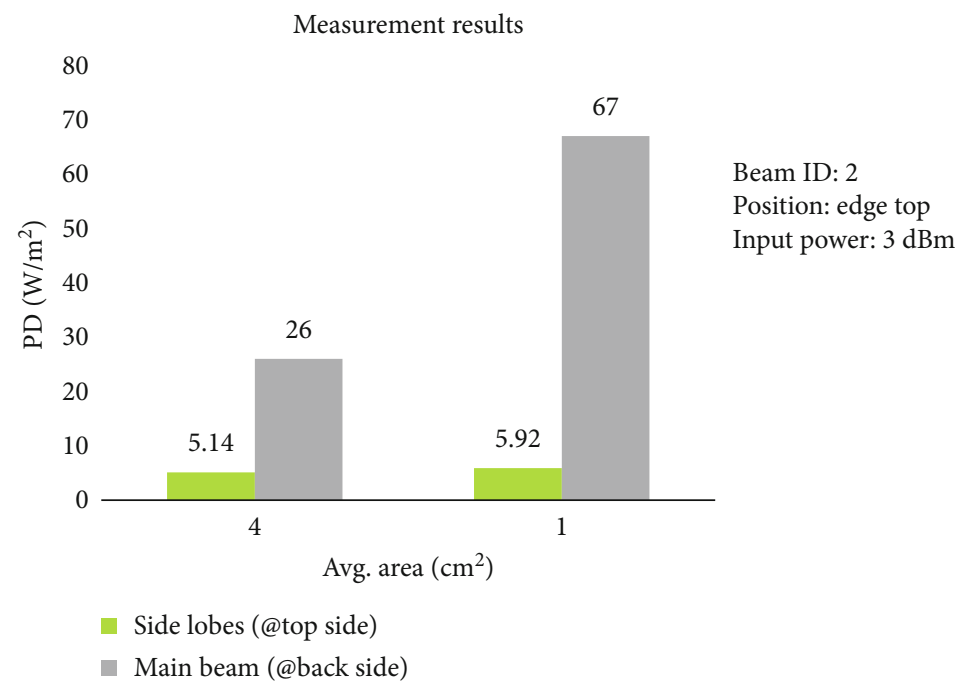

FIGURE 21: Measurement results in a nonpeak direction for beam 2. 


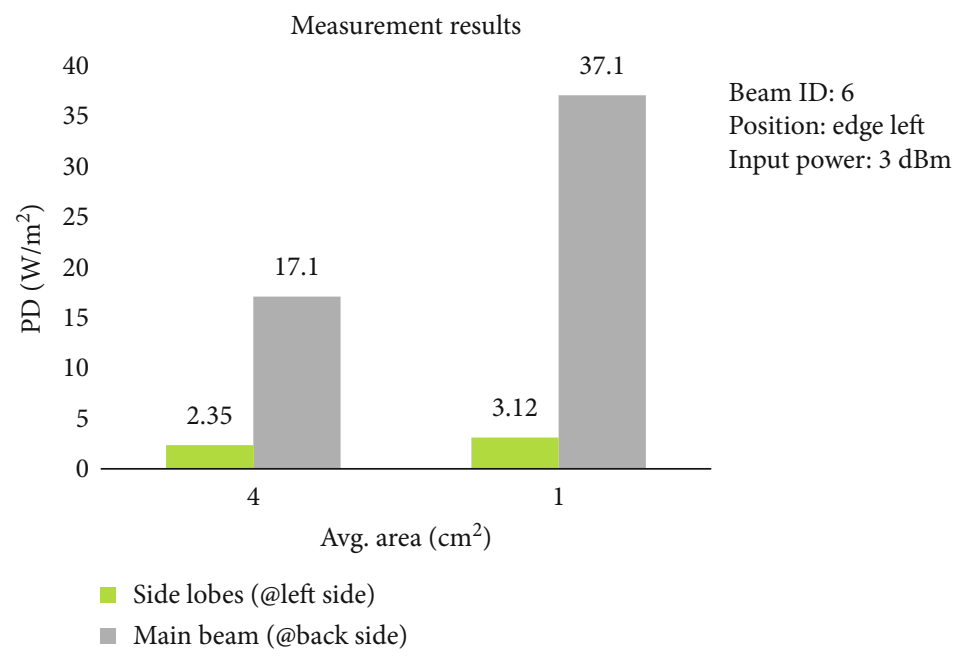

FIGURE 22: Measurement results in a nonpeak direction for beam 6.

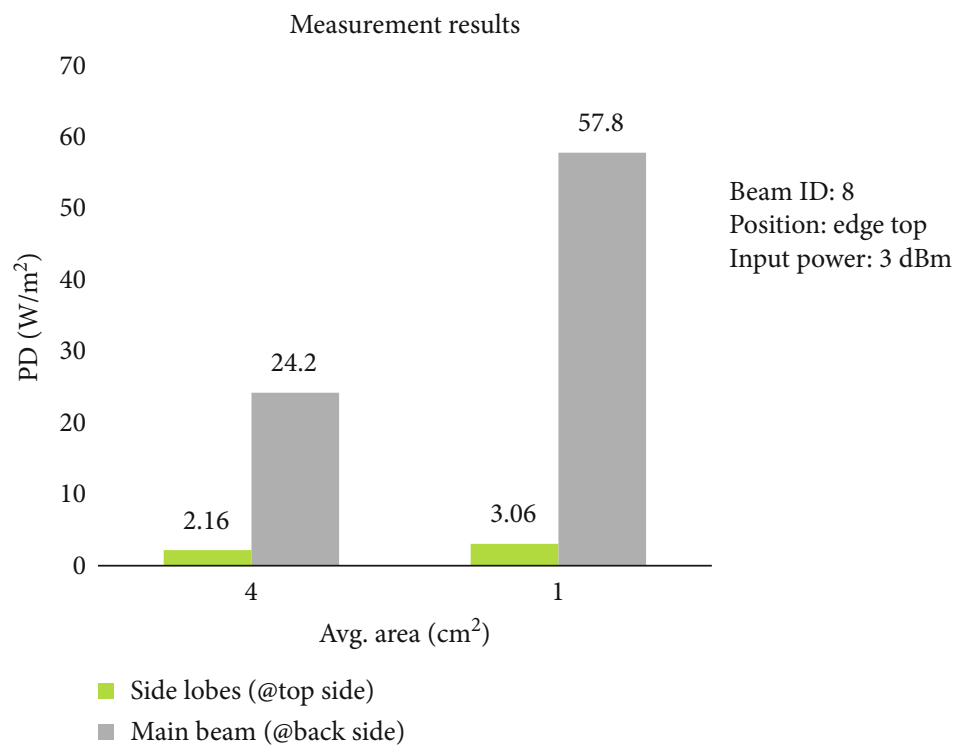

FIGURE 23: Measurement results in a nonpeak direction for beam 8 .

results. The insertion loss difference of each panel has also been considered for the simulation and measurement results normalization.

\subsection{Analysis}

(1) The deviations between PD simulation and measurement results of the selected $2 \times 2$ patch antenna array beams were verified to be within $\pm 2.4 \mathrm{~dB}$

(2) From simulation and measurement results, we could check the correlation of power density variation. The PD trends between simulation and measurement may not ideal one-to-one correspondence. But the top four highest beams from PD simulation at most can still cover the worst case of PD measurement

Figure 24 shows the cross-sectional power density plot of the normal and offset beams. The PD measurement results show that the normal beam (e.g., beam 5) is the worst case in the panel. While in the simulation, the PD simulation result of the offset beam (e.g., beam 4) is slightly higher than the normal beam.

In the near field simulation region, there may be a case where the offset beam PD is slightly higher than the normal beam due to the influence of the shell and the close distance. The different array elements may occur in the beam aggregation region. It is probably because the truncated DUT model is used, and the surrounding shells are not accurately modeled. 
TABLE 1: PD simulation and measurement results comparison.

\begin{tabular}{|c|c|c|c|c|c|}
\hline \multirow[b]{2}{*}{ Test condition } & \multirow{2}{*}{$\begin{array}{l}\text { Beam } \\
\text { ID }\end{array}$} & \multirow{2}{*}{$\begin{array}{l}\text { Panel insertion loss } \\
\qquad(\mathrm{dB})\end{array}$} & \multicolumn{2}{|c|}{ Normalized results } & \multirow{2}{*}{$\begin{array}{l}\text { Deviation } \\
\quad(\mathrm{dB})\end{array}$} \\
\hline & & & $\begin{array}{l}\text { Simulation } \\
\left(\mathrm{W} / \mathrm{m}^{2}\right)\end{array}$ & $\begin{array}{l}\text { Measurement } \\
\left(\mathrm{W} / \mathrm{m}^{2}\right)\end{array}$ & \\
\hline \multirow{18}{*}{$2 \times 2$ patch antenna array, back side, $5 \mathrm{~mm}$} & 1 & 0.6 & 31.72 & 25.6 & -0.93 \\
\hline & 2 & 0.6 & 25.71 & 26 & 0.05 \\
\hline & 3 & 0.6 & 23.58 & 22 & -0.3 \\
\hline & 4 & 0.6 & 34.37 & 25 & -1.38 \\
\hline & 5 & 0.6 & 30.22 & 34.2 & 0.54 \\
\hline & 6 & 0.6 & 25.88 & 17.1 & -1.8 \\
\hline & 7 & 0.6 & 31.72 & 19 & -2.23 \\
\hline & 8 & 0.6 & 25.69 & 24.2 & -0.26 \\
\hline & 9 & 0.6 & 23.68 & 13.8 & -2.35 \\
\hline & 10 & 1.45 & 23.22 & 21.4 & -0.35 \\
\hline & 11 & 1.45 & 24.99 & 22.3 & -0.49 \\
\hline & 12 & 1.45 & 23.17 & 18.1 & -1.07 \\
\hline & 13 & 1.45 & 29.89 & 25.4 & -0.71 \\
\hline & 14 & 1.45 & 31.06 & 28.8 & -0.33 \\
\hline & 15 & 1.45 & 31.25 & 28.4 & -0.42 \\
\hline & 16 & 1.45 & 23.42 & 16.2 & -1.6 \\
\hline & 17 & 1.45 & 25.76 & 27.2 & 0.24 \\
\hline & 18 & 1.45 & 23.22 & 20.3 & -0.58 \\
\hline
\end{tabular}

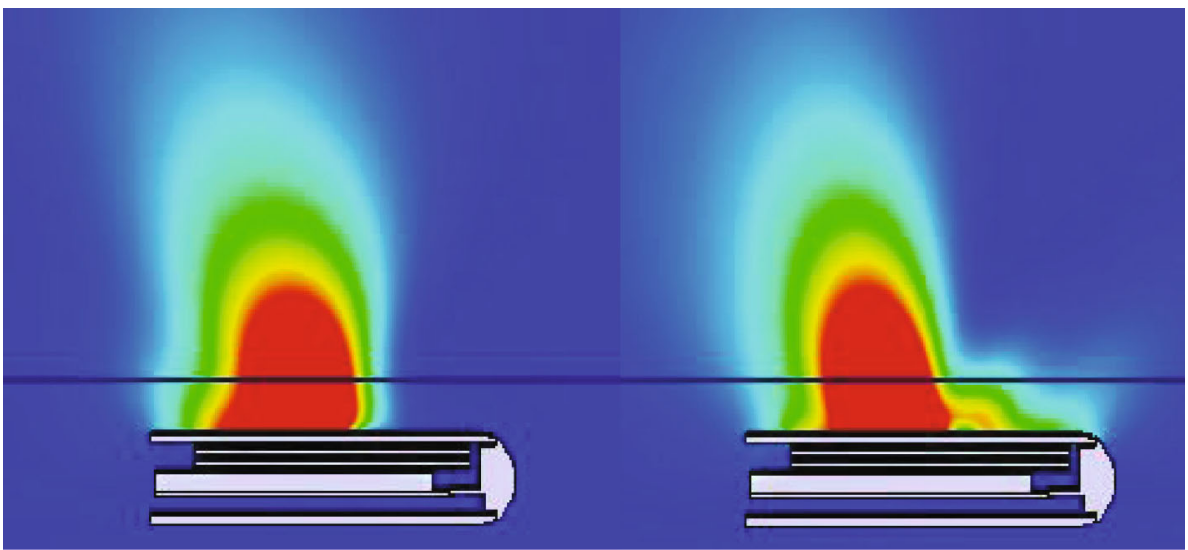

Figure 24: Cross-sectional power density plot of the normal and offset beams.

\section{Conclusion}

For mmWave array antennas, when practical, the combination of numerical simulation and measurements to demonstrate the power density compliance is a practicable assessment approach and a considerable reduction in the measurement period. As

(1) from power density measurement results of multiple factors (different beam configurations, test positions, input power levels, duty cycles, main lobe and side lobe beam directions, etc.), we could check the correlation of power density variation. It would be a good reference when developing IEC standard
(2) from power density simulation and measurement results comparison, it is worth to consider the power density results from the simulation in term of showing compliance

\section{Data Availability}

The measurement and simulation data used to support the findings of this study are included within the article.

\section{Conflicts of Interest}

The authors declare that they have no conflicts of interest. 


\section{References}

[1] K. R. Foster, S. Kodera, and A. Hirata, " 5 G communication systems and radiofrequency exposure limits," IEEE Future Networks Tech Focus, vol. 3, no. 2, 2019.

[2] T. Wu, T. S. Rappaport, and C. M. Collins, "The human body and millimeter-wave wireless communication systems: interactions and implications," in 2015 IEEE International Conference on Communications (ICC), pp. 2423-2429, London UK, 2015.

[3] American National Standards Institute, Safety Levels with Respect to Human Exposure to Radio Frequency Electromagnetic Fields, $300 \mathrm{kHz}$ to $100 \mathrm{GHz}$, vol. 10016, The Institute of Electrical and Electronic Engineering New York, 1982. 